\title{
La libertad como poder semoviente en Émilie Du Châtelet: reflexiones tempranas
}

\author{
Natalia Zorrilla ${ }^{1}$
}

Resumen: El objetivo de este artículo es examinar algunas continuidades y discontinuidades entre un texto de Émilie Du Châtelet titulado De la liberté, su epistolario y sus Institutions de physique en torno al tratamiento que la filósofa realiza de la temática de la libertad como poder semoviente. La hipótesis que propongo en este trabajo es que ciertos problemas filosóficos que Du Châtelet se plantea tempranamente se resuelven (o se disuelven) en las Institutions de physique. Nos referimos puntualmente a: 1. la presunta contradicción entre la idea del poder semoviente del cuerpo propio y la conservación de la cantidad de fuerza en el universo y 2. la presunta falta de garantías del funcionamiento veraz de nuestras facultades cognitivas (mediante las cuales constatamos que somos libres).

Palabras-clave: Émilie Du Châtelet - Libertad - De la liberté - Siglo XVIII - Fatalisme - Ilustración

\section{Freedom as a self-moving power in Émilie Du Châtelet: early reflections}

\begin{abstract}
The aim of this article is to examine continuities and discontinuities between Émilie Du Châtelet's essay De la liberté, her correspondence, and her Institutions de physique regarding the topic of liberty as a self-moving power. I argue that certain philosophical problems that she considers in $D e$ la liberté and in her letters are resolved (or dissolved) in her Institutions de physique, namely: 1. the alleged contradiction between the idea of a self-moving power and the conservation of the quantity of force in the universe, and 2. the alleged lack of guarantee of the veraciousness of our cognitive faculties (through which we perceive our liberty).

Keywords: Émilie Du Châtelet - Liberty - De la liberté - Eighteenth Century - Fatalisme Enlightenment
\end{abstract}

\footnotetext{
${ }^{1}$ Becaria postdoctoral del Consejo Nacional de Investigaciones Científicas y Técnicas (CONICET), Instituto de Filosofía Dr. Alejandro Korn - Universidad de Buenos Aires (UBA). Investigadora asociada del Centro de Investigaciones Filosóficas (CIF), Argentina. Mail: n.zorrilla@conicet.gov.ar
} 


\section{Introducción ${ }^{2}$}

En las últimas décadas, fruto de los avances de la revisión feminista de la historiografía aplicada a la historia de la filosofía, se ha producido un marcado viraje en la forma en que se aborda la figura de Émilie Du Châtelet (1706-1749). Gracias a esta revisión, se han comenzado a ponderar los logros intelectuales de esta filósofa por mérito propio. En el presente artículo buscamos contribuir a la recuperación de la obra de esta pensadora, ocupándonos principalmente de un breve ensayo de Du Châtelet titulado De la liberté (en adelante DL). Intentaremos mostrar, a través del análisis del DL, el proceso de reflexión de Du Châtelet respecto de ciertos problemas filosóficos que ella se plantea tempranamente cuya resolución (o disolución) se reflejará en la redacción de las Institutions de physique (1740) (en adelante IP), usualmente considerada la obra magna de la pensadora. Nos referimos puntualmente a: 1. la presunta contradicción entre la idea del poder semoviente del cuerpo propio (poder en que consistiría nuestra libertad) y la conservación de la cantidad de fuerza en el universo y 2. la presunta falta de garantías del funcionamiento veraz de nuestras facultades cognitivas (mediante las cuales constatamos que somos libres).

En primer lugar, ofreceremos algunas precisiones acerca de los avatares del descubrimiento, la publicación y la atribución de autoría del DL. En segundo lugar, abordaremos el carácter inicialmente problemático de la concepción de Du Châtelet de la libertad como poder semoviente en su epistolario. Intentaremos mostrar cómo esta problemática se disuelve durante el proceso de redacción de las IP. En tercer lugar, examinaremos la contienda entre Du Châtelet y el fatalismo en DL, estudiando la reconstrucción que la filósofa realiza de esta posición rival, particularmente, la formulación de objeciones fatalistas a la libertad como argumentos escépticos (que evocan aquellos que Descartes plantea en la Primera Meditación y en la primera parte de sus Principios de Filosofía). Estos argumentos socavan nuestra confianza en la veracidad de nuestras facultades cognitivas en relación con la percatación del sentimiento interno de libertad. Finalmente, estudiaremos las críticas que Du Châtelet realiza a Descartes y cómo separarse de este pensador le sirve para blindarse contra los argumentos escépticos que él pondera en su obra.

\section{De la liberté: descubrimiento y autoría}

En 1947, Ira Wade publica algunos estudios sobre Voltaire junto con ciertos papeles inéditos de Émilie Du Châtelet que descubre en la "Biblioteca Voltaire" (Voltaire Library) en la actual Biblioteca Nacional Rusa (anteriormente Biblioteca Imperial de San Petersburgo $)^{3}$. Esta importante colección de libros y manuscritos que formaban parte de la biblioteca personal de Voltaire fue adquirida por Catalina II, Emperatriz de Rusia, poco después de la muerte del filósofo y luego trasladada a Rusia. Entre los materiales publicados por Wade se encuentra un breve tratado titulado "Capítulo V: De la libertad" (el DL) que él

\footnotetext{
${ }^{2}$ Este trabajo surge de una presentación realizada en el I Encontro Argentina-Brasil sobre Estudos do Século XVIII/I Encuentro Argentina-Brasil sobre Estudios del Siglo XVIII en octubre del 2020. Agradezco a los/as colegas por sus comentarios y a Susana Seguin por facilitarme distintos materiales para la composición de este artículo.

3 WADE, I. Studies on Voltaire. With Some Unpublished Papers of Mme du Chattelet. Princeton: Princeton Univ. Press, 1947.
} 
atribuye a Voltaire. Wade entiende que este breve ensayo fue un borrador que Voltaire cortó, editó y re-escribió de donde habría surgido el actual capítulo VII del Traité de métaphysique. Wade se apoya en el intercambio epistolar entre Voltaire y quien en 1740 se convertiría en Federico II de Prusia ${ }^{4}$, dado que en este intercambio epistolar, Voltaire le envía a Federico un "extracto de un capítulo sobre la libertad" como un texto de su autoría.

Linda Gardiner Janik ${ }^{5}$ desarrolla una hipótesis contraria a la de Wade. Ella sostiene que el DL era el borrador de un capítulo de la primera versión de las IP escrita entre 1737 y 1738 que luego Du Châtelet revisa y aumenta radicalmente. Una de las evidencias que apoyan esta tesis puede hallarse en el inventario de los manuscritos de Voltaire conservados en Rusia redactado por Fernand Caussy. Allí puede constatarse que el manuscrito del DL está catalogado como perteneciente a los papeles de Mme Du Châtelet ${ }^{6}$. A su vez, contiene notas de Voltaire que identifican a Du Châtelet como su autora ${ }^{7}$ y referencias a otros parágrafos que, según Gardiner Janik, encajan dentro de la primera versión de las IP.

¿Cómo explica Gardiner Janik entonces el dato de que Voltaire le habría enviado a Federico el extracto de un capítulo sobre la libertad identificándose como su autor? Ella sugiere que Voltaire podría haber sido deshonesto con Federico en este punto, adjudicándose un texto que no era suyo. Gardiner Janik argumenta que, como Du Châtelet no tenía mucha simpatía por Federico ${ }^{8}$, ella podría incluso haber disfrutado irónicamente viéndolo elogiar una obra que atribuía a Voltaire cuando en realidad el texto había sido escrito por ella. Esto también explicaría en parte por qué Du Châtelet no habría incluido el DL en las IP finalmente: para evitar ser acusada de plagio?.

\section{La libertad como poder semoviente: epístolas}

El interés de Du Châtelet por el problema de la libertad y sus limitaciones y alcances se expresa claramente en su epistolario. La filósofa encuentra una aparente contradicción entre dos tesis que ella misma defiende: por un lado, la idea de que existe la libertad humana, entendida como el poder de iniciar el movimiento del cuerpo propio con el fin de realizar alguna acción, y, por otro, la premisa de que la cantidad de fuerza permanece idéntica en el universo. En una carta a Maupertuis, datada el 30 de abril de 1738, ella confiesa lo siguiente:

La única cosa que me preocupa actualmente es la libertad, porque en definitiva me creo libre y no sé si esta cantidad de fuerzas, siempre la misma en el universo, no destruye la libertad. Comenzar el movimiento, ¿no es producir en la naturaleza una fuerza que no existía [previamente]? Pero si no

\footnotetext{
${ }^{4}$ WADE, Studies, pp. 82-87.

5 Véase GARDINER JANIK, "Searching for the Metaphysics of Science: The Structure and Composition of Madame du Châtelet's Institutions de physique, 1737-1740”.

${ }^{6}$ Véase CAUSSY, Inventaire des manuscrits de la Bibliothèque de Voltaire conservés a la Bibliothèque Impériale de SaintPétersbourg, p. 43.

${ }^{7}$ Voltaire y Du Châtelet forjaron un estrecho vínculo sentimental y de colaboración intelectual desde 1734 durante la estadía de Voltaire en Cirey. Véase ZINSSER, La Dame d'Esprit: A Biography of the Marquise Du Châtelet, pp. 115-221.

${ }^{8}$ El intercambio epistolar entre Voltaire y Federico revela un tono de flirteo y seducción entre ambos, con reproches por parte del segundo al primero por serle infiel con una "coqueta" (que sería Du Châtelet). Para ahondar en el intercambio epistolar "libertino" entre ambos, véase MASON, Voltaire, pp. 67-70.

${ }^{9}$ GARDINER JANIK, “Searching for the Metaphysics of Science...”, p. 110.
} 
tenemos el poder de comenzar el movimiento, no somos libres. Le suplico me aclare este asunto $^{10}$.

En su exploración del manuscrito autógrafo de las IP de la Biblioteca Nacional Francesa de 1737, Rey llama la atención sobre un parágrafo (\$720) que no habría sido incluido en la versión impresa de las IP. Allí Du Châtelet finalmente resuelve este dilema entre la libertad humana y la conservación de la cantidad de fuerza en el universo, estableciendo una distinción entre cantidad de fuerza y cantidad de movimiento. Rey transcribe una anotación marginal que resume el contenido del parágrafo:

La conservación de una cantidad igual de fuerza en nuestro universo parece en principio incumbir a nuestra libertad, pero únicamente cuando confundimos la cantidad de movimiento con la cantidad de fuerza. Si somos libres, nuestra voluntad produce sin duda movimiento, porque si depende de mí caminar o quedarme sentada, es seguro que, al caminar, produciré un movimiento que no habría producido si me hubiese quedado en mi silla. Pero ¿es necesario para esto que yo produzca fuerza? No, sin duda. Yo puedo ser libre al emplear la cantidad de fuerza que se encuentra en mis nervios y en mi sangre, sin que por eso mi voluntad pueda crear fuerza ${ }^{11}$.

De esta manera, concluye la filósofa, la libertad, entendida como poder semoviente respecto del cuerpo propio, no es incompatible con el principio de la conservación de la cantidad de fuerza en el universo. Podemos conectar estas observaciones con una de las primeras anotaciones con las que nos encontramos en el manuscrito de DL, la cual habría sido "sustituida" (o eliminada), de acuerdo con las indicaciones de la transcripción de Wade. La nota dice: "No examino aquí si la opinión que quiere que la cantidad de fuerza permanezca la misma en el universo es tan contraria a la libertad como aquella [que afirma] una igual cantidad de movimiento ${ }^{12}$. Véase sobre eso el capítulo VIII”"13.

\footnotetext{
10 Todas las traducciones de las citas de textos de Du Châtelet son propias. "La seule chose qui m'embarrasse à présent, c'est la liberté, car enfin je me crois libre et je ne sais si cette quantité de forces toujours la même dans l'univers ne détruit point la liberté. Commencer le mouvement, n'est-ce pas produire dans la nature une force qui n'existait pas? Or si nous n'avons pas le pouvoir de commencer le mouvement nous ne sommes point libres. Je vous supplie de m'éclairer sur cet article" (Carta a Maupertuis, 30 de abril de 1738. DU CHÂTELET, Les Lettres de la Marquise Du Châtelet, vol. I, p. 220). Ante una respuesta insatisfactoria de Maupertuis, Du Châtelet vuelve sobre esta cuestión en una carta del 9 de mayo de 1738. Véase DU CHÂTELET, Les Lettres de la Marquise Du Châtelet, vol. I, p. 226.

11 "La conservation d'une quantité égale de force dans notre univers parait d'abord intéresser notre liberté, mais ce n'est que lorsque l'on confond la quantité du mouvement et la quantité de la force, si nous sommes libres, notre volonté produit sans doute du mouvement, car s'il dépend de moi de me promener ou de rester assis, il est certain qu'en me promenant, je produirai un mouvement que je n'eusse pas produit si j'étais resté sur ma chaise, mais est-il nécessaire pour cela que je produise de la force, non sans doute, je puis être libre dans l'emploi de la quantité de force qui se trouve dans mes nerfs et dans mon sang, sans que pour cela ma volonté puisse créer de la force" (DU CHÂTELET, Institutions de Physique [manuscrito], foja 370, citado en REY, "La Minerve vient de faire sa physique", p. 240).

${ }^{12}$ La anotación marginal al primer párrafo del cuerpo principal del texto afirma "Dieu a reellement donne a l'homme le pouvoir soi-mouvant"; "Dios le ha dado realmente al hombre el poder semoviente" (WADE, Studies, p. 92).

13 “Je n'examine point ici si l'opinion qui veut que la quantité de force reste la meme dans l'univers est aussi contraire a la liberté que celle d'une egale quantité de mouvement, voyez sur cela ch 8" (WADE, Studies, p. 92).
} 
Se observa así la continuidad que existe entre las preocupaciones filosóficas de $\mathrm{Du}$ Châtelet, sobre las que se expide en las obras de este período temprano. Más aún, estas preocupaciones son de suma importancia para ella debido a sus implicancias morales: según sugiere la autora, si existiese una necesidad de orden metafísico en las acciones humanas, esto redundaría en una exención de responsabilidad moral generalizada.

\section{La contienda entre Émilie Du Châtelet y el fatalismo en De la liberté}

En DL, Du Châtelet también define la libertad como poder moverse o no moverse de acuerdo con la elección de nuestra mente, agregando una segunda acepción: libertad también es poder pensar o no pensar en algo de acuerdo con la elección de nuestra mente ${ }^{14}$. El carácter polémico del DL define su estructura: en tanto su objetivo es vencer a ciertos interlocutores o interlocutoras "fatalistes", nuestra autora reconstruye las principales objeciones a la idea de que somos libres y luego se aplica a refutarlas.

A principios del siglo XVIII, el término "fatalisme", que aquí traduciremos como "fatalismo", aparecía en tratados y comentarios varios con distintos sentidos. Intérpretes como Paillard ${ }^{15}$ sugieren que, durante el Siglo de las Luces, "fatalismo" a menudo se tomaba equívocamente como sinónimo de lo que contemporáneamente entendemos por "determinismo" y también por "necesitarismo"16. En líneas generales, cuando el fatalismo era asociado a cierto spinozismo y/o al materialismo, equivalía a defender la tesis de que todo existe necesariamente y/o que todos los fenómenos de la naturaleza están causalmente determinados, incluidas las acciones humanas. En estos casos, el término "fataliste" funcionaba frecuentemente como una acusación. Sus detractores querían demostrar que quienes defendían el "sistema de la fatalidad" impedían la atribución de responsabilidad moral a los agentes y promovían con ello la resignación y la inmoralidad. También existía en aquella época otro tipo de fatalismo: el teólogico, evocado en DL en su formulación "optimista" surgida de la obra de Pope y Leibniz ${ }^{17}$.

Du Châtelet distingue cuatro objeciones fatalistas principales; en nuestro artículo, nos concentraremos solamente en las dos primeras. De acuerdo con nuestra interpretación, en ellas, Du Châtelet reconstruye la posición de un fatalismo del primer tipo mencionado anteriormente, el cual utiliza argumentos escépticos para socavar la tesis de la existencia del libre arbitrio. Nuestra hipótesis respecto de la formulación que Du Châtelet realiza de las dos primeras objeciones fatalistas es que la filósofa emularía en ellas el movimiento de la duda metódica cartesiana, reversionando los argumentos escépticos de Descartes en la Primera Meditación (correspondientes a los parágrafos $\ 4-5$ de la primera parte de los Principios de la filosofi $\left.a^{18}\right)$ para procurar adaptarlos a una formulación fatalista. Mientras que la primera

\footnotetext{
${ }^{14}$ En "Du Châtelet on freedom, self-motion, and moral necessity", Julia Jorati argumenta que, basándose en Locke y en algunas ideas de Clarke, Du Châtelet defiende una posición compatibilista, buscando refutar la existencia de la necesidad en sentido físico pero admitiendo la necesidad en sentido moral en lo que concierne a las acciones humanas.

15 PAILLARD, "Entre science et métaphysique: le problème du fatalisme dans la philosophie de Voltaire", pp. 211-212.

16 Para un análisis contemporáneo del fatalismo, véase BERNSTEIN, "Fatalism".

17 Véase ZANCONATO, La dispute du fatalisme en France: 1730-1760.

${ }^{18}$ AT 8: 5-6. Tenemos evidencia de que Du Châtelet leyó y comentó esta obra, ya que se conservan actualmente algunas notas de sus cuadernos referidas a los Principios de Descartes. Por esta razón, indicaremos en nuestra reconstrucción los parágrafos correspondientes de los Principios de Descartes. Véase la edición digital de estas
} 
objeción fatalista de DL exhibe rasgos característicos del argumento del sueño (combinado con el de los sentidos), la segunda evoca el argumento del Dios engañador-aunque, como veremos, también toma en cuenta implicancias de los argumentos escépticos anteriores. La peculiaridad del planteo de la filósofa residiría entonces en que, para articular su posición rival, ella yuxtapone fatalismo y pirronismo ${ }^{19}$ : le adjudica al primero las armas del segundo.

A través de estas dos objeciones, Du Châtelet se enfrenta al problema de cómo podemos estar seguros/as de que ese sentimiento interior de libertad que experimentamos no proviene de la falta de aplicación o discernimiento de nuestras facultades o de un engaño sistemático al que estaríamos siendo sometidos/as. Según la pensadora, este sentimiento interior funciona como un presupuesto necesario de nuestras acciones y decisiones. No solamente sentimos que somos libres al movernos en una u otra dirección o evocando algún recuerdo o alguna fantasía imaginaria en nuestra mente, sino que además, al deliberar, decidir y realizar una acción, presuponemos que está en nuestro poder ejecutarla.

Antes de pasar al análisis puntual de las objeciones fatalistas en cuestión, es menester aclarar que, a lo largo de su obra, Descartes defiende la existencia del libre arbitrio y la infinitud de la voluntad. No obstante, al ser nuestro entendimiento finito, podemos errar según este pensador. Erramos en nuestros juicios cuando no nos abstenemos de pronunciarnos sobre cuestiones que no se nos presentan con claridad y distinción a nuestro espíritu $^{20}$. El error entonces no puede darse en lo que el sujeto percibe que percibe ${ }^{21}$ sino en lo que juzga que sea el objeto percibido ${ }^{22}$.

Así, Descartes sostiene que el libre albedrío es una evidencia que se presenta clara y distintamente a todos los sujetos pensantes, una noción primera e innata ${ }^{23}$. En cambio, si bien Du Châtelet también defiende la existencia de un sentimiento interior de nuestra libertad, ella parece estar advertida sobre los peligros del posible corolario pirrónico del solipsismo cartesiano ${ }^{24}$ respecto de este punto: puede que yo me sienta libre pero ¿cómo

notas, titulada "Three notebook leaves on Descartes, Newton, Fontenelle", realizada por Andrew BROWN, Ulla KÖLVING y Stefanie ERTZ (Center for the History of Women Philosophers and Scientists, University of Paderborn, en cooperación con la National Library of Russia, Saint Petersburg, y el Centre international d'étude du XVIIIe siècle, Ferney-Voltaire). Cf. https://historyofwomenphilosophers.org/stp/documents

${ }^{19}$ Las principales figuras del escepticismo pirrónico en la Antigüedad fueron Enesidemo, Pirrón y Sexto Empírico (autor de las Hipotiposis pirrónicas). Los pirrónicos utilizaban una serie de tropos en contra de las pretensiones dogmáticas de aquellos/as que afirmaban que se puede conocer la verdad, esto es: cómo son las cosas en sí mismas. A diferencia de los escépticos académicos, los pirrónicos no sostienen que la verdad es incognoscible sino que intentan lograr mediante estos tropos la suspensión del juicio o epoché. Se guían por las apariencias buscando la ataraxia o imperturbabilidad. Para una aproximación al pirronismo publicada algunos años antes de la redacción de DL, véase CROUSAZ, Examen du pyrrbonisme ancien et moderne (1733).

${ }^{20}$ DESCARTES, Principios I $\int 33$ (AT 8: 17-18). En Principios I §6 (AT 8: 6) es la noción de libertad la que, de hecho, le permite resolver la duda hiperbólica y llegar al cogito ergo sum: aunque seamos sometidos/as a un engaño generalizado, siempre tendremos la libertad de abstenernos de creer en algo que se nos presenta como incierto o dudoso. En Meditaciones, el tratamiento del tema del libre arbitrio se da en la Cuarta Meditación, posteriormente a haber probado la existencia de Dios, garante de nuestros conocimientos.

${ }^{21}$ Se suele atribuir a Descartes la llamada tesis de la transparencia de la mente, la cual implica que todos los estados mentales son conscientes y que tenemos un conocimiento infalible de ellos. Algunos/as comentadores como Hatfield discuten esta descripción de la filosofía de Descartes. Véase HATFIELD, "Transparency of Mind: The Contributions of Descartes, Leibniz, and Berkeley to the Genesis of the Modern Subject".

22 DESCARTES, Principios I $\$ 66$ (AT 8: 33-34).

23 DESCARTES, Principios I $\$ 39$ (AT 8: 19-20).

24 Siguiendo a Charles, si bien actualmente utilizamos el término "solipsismo", los/as pensadores/as ilustrados/as franceses/as a menudo usaban los términos "idéalisme" o "égoïsme" para referirse a esta misma tesis. CHARLES, "O solipsismo como forma extrema de ceticismo no Século das Luzes”, p. 17. Consúltese este artículo para ahondar acerca del estrecho vínculo entre solipsismo y escepticismo durante el siglo XVIII. 
puedo tener la certeza de que otra persona también lo sienta respecto de sî́ ${ }^{25}$ Las objeciones fatalistas pueden ser "seductoras" 26 , según la autora, es decir, persuasivas. De aquí que ella se aboque a considerar la posición de quienes desestiman este sentimiento de libertad.

Como veremos más adelante en nuestro análisis, ya en DL podemos constatar que para Du Châtelet resultaría problemático admitir que un fundamento epistémico puramente subjetivo del conocimiento filosófico-científico (como el que ella considera que propondría Descartes) pueda tener validez universal. Por eso, la filósofa entiende que debe enfrentarse al desafío escéptico y solipsista legado por Descartes. En consecuencia, en DL imagina un interlocutor fatalista que utiliza contra Descartes (y en general contra quienes afirman la evidencia del sentimiento de libertad al actuar y pensar) las mismas armas escépticas que el autor de las Meditaciones metafísicas emplea para socavar (aunque más no sea momentáneamente) nuestra confianza en nuestras facultades cognitivas.

Cuando Descartes se propone encontrar las verdades indubitables, fundamentos de las ciencias, aplica para ello la duda metódica: se propone poner en duda todas sus antiguas opiniones. Para lograrlo, no las examina una por una (sería una tarea infinita) sino que evalúa distintas posibles fuentes y facultades de conocimiento sometiéndolas a argumentos escépticos varios. El objetivo de Descartes es rechazar como falsas todas aquellas cosas que parezcan dudosas, aunque solo en el marco de la búsqueda filosófica de verdades indubitables (no en la práctica de la vida).

Descartes hace un uso metodológico o instrumental del escepticismo y pretende de esta manera intervenir en lo que Popkin denomina la "crise pyrrhonienne". Popkin indica que esta crisis se suscita en gran medida a causa de contiendas religiosas, específicamente la Reforma, la cual habría puesto de manifiesto la importancia de la problemática del criterio de verdad. Esta problemática se reprodujo en el campo de la filosofía, fomentada por la recuperación durante los siglos XV y XVI de la obra de Sexto Empírico por parte de los llamados “nouveaux pyrrhoniens". Según el historiador, el intento de Descartes de resolver los tropos escépticos y en última instancia de "salvar al conocimiento humano destruyendo al escepticismo" fue un "heroico fracaso"27, ya que durante los siglos XVII y XVIII el pirronismo siguió prosperando a pesar suyo y contra su filosofía, a partir de distintas lecturas de su obra.

Así, Descartes lega a la posteridad mediante estos tropos escépticos ciertos problemas a resolver con los que el pirronismo buscaría sembrar una duda generalizada. Desde nuestro punto de vista, el objetivo de Du Châtelet es desarticular estos argumentos escépticos que Descartes se había planteado pero bajo una nueva formulación, es decir, orientados al problema de la libertad. El DL sería entonces un texto de transición, que enlaza las preocupaciones filosóficas tempranas de Du Châtelet acerca de la libertad que se manifiestan en su epistolario con su rechazo del subjetivismo en las IP. En DL, ambos puntos se unen en el tratamiento que Du Châtelet le da al fatalismo.

\footnotetext{
${ }^{25}$ Mayores precisiones acerca de este tema pueden encontrarse en la sección "Du Châtelet contra Descartes en Institutions de Physique (o cómo blindarse contra ataques pirrónicos)".

${ }^{26}$ WADE, Studies, p. 93.

${ }^{27}$ POPKIN, The history of scepticism from Savonarola to Bayle, p. 80 .
} 


\section{El primer argumento}

La primera objeción contra la libertad que Du Châtelet considera aduce que si sostenemos que existe un sentimiento interno o conciencia de que somos libres, es por falta de atención a lo que pasa en nosotros/as. En efecto, la voz fatalista imaginada por Du Châtelet argumenta aquí que "cuando atendemos reflexivamente a las causas de nuestras acciones, encontramos por el contrario que ellas están siempre determinadas necesariamente" 28 .

Se ofrecen adicionalmente dos ejemplos para reforzar este punto de vista fatalista: los movimientos involuntarios que ocurren en nuestro cuerpo (por ejemplo: la circulación de la sangre) y los arrebatos pasionales que nublan nuestro juicio y nos llevan a realizar acciones desaprobadas por nuestra razón. En cuanto a este último, según la objeción fatalista, se puede ser esclavo/a de pasiones violentas como la ira pero también de pasiones suaves cuando una no se percata que está siendo "conducida" por ellas. El fatalista busca arrojar un manto de sospecha respecto de este sentimiento interno de libertad y sugerir que nunca se puede saber si una está siendo influenciada por tal o cual pasión o si está actuando “autónomamente". O bien, tal como lo presenta Du Châtelet, el fatalista estaría razonando de la siguiente manera: debido a que a veces nos encontramos bajo el influjo de las pasiones (lo que puede hacernos actuar de formas que la razón desaprueba), debemos suponer que siempre somos (o podríamos estar siendo) esclavos/as de las pasiones sin saberlo.

En Principios I \$4, Descartes pretende abordar "por qué podemos dudar de las cosas sensibles". Descartes adiciona aquí el argumento del sueño al argumento de los sentidos. Los sentidos yerran algunas veces, por lo tanto, por aplicación de la duda metódica, no nos podemos fiar de ellos para encontrar certezas absolutas. Asimismo, en nuestros sueños, "nos parece sentir o imaginar innumerables cosas que no hay en ninguna parte, y al que duda de esta manera [acerca de si efectivamente existen los objetos exteriores que percibe mientras sueña] no se le presenta ningún signo por el cual pueda distinguir el sueño de la vigilia" ${ }^{29}$. La aplicación de la duda metódica a la facultad de los sentidos y de la imaginación en las Meditaciones y en los Principios da como resultado un razonamiento análogo al que Du Châtelet le atribuye al fatalista en DL: si los sentidos alguna vez me han engañado, debo suponer que siempre me pueden engañar. Si alguna vez me he engañado acerca de la existencia de los cuerpos que percibo, debo suponer que siempre puedo estar engañándome. De la misma manera, si alguna vez me he engañado acerca de mi imperio sobre mi cuerpo o mis acciones, debo suponer que siempre podría estar engañándome. Tal engaño podría suceder, por ejemplo, mientras esté soñando o bien mientras esté actuando bajo el influjo de las pasiones sin darme cuenta.

El fatalista presupone así que las pasiones del alma (cuya causa no se especifica) pueden ser naturalmente engañosas en tanto no nos percatemos de que están afectando nuestra alma y obliterando nuestra racionalidad. Desde el punto de vista cartesiano, en cambio, las pasiones del alma son percepciones "que se refieren solo al alma, (...) cuyos efectos sentimos en el alma misma y de las que, por lo general, no conocemos ninguna causa

\footnotetext{
28 "Lorsque nous faisons [au contraire] une attention réfléchie sur les causes de nos actions, nous trouvons au contraire qu'elles sont toujours déterminées nécessairement" (WADE, Studies, p. 93). Entre corchetes se transcriben, siguiendo a Wade, las frases que fueron borradas (deleted) del manuscrito.

${ }^{29}$ DESCARTES, Principios I \$4, p. 8 (AT 8: 6).
} 
inmediata con la que podamos relacionarlas"30. Para Descartes, ellas cumplen la función de disponer al alma "a querer cosas que la naturaleza dicta que nos son útiles y a persistir en esa voluntad"31. Según Descartes, pueden ser reguladas, aunque indirecta y mediatamente, a través de nuestra voluntad ${ }^{32}$. Por lo tanto, si bien conllevan cierta oscuridad en sí debido a la indeterminación de su origen, no son por naturaleza engañosas—somos conscientes de su influjo. Para el fatalista al que alude Du Châtelet, por el contrario, las pasiones pueden no ser detectadas por quienes las padecen (al menos momentáneamente).

En efecto, cuando Du Châtelet, al reconstruir la objeción fatalista, describe cómo sería ese engaño de vivir bajo el influjo de las pasiones sin poder actuar racional y autónomamente, hace uso de una metáfora: el esclavo que no advierte el peso de sus cadenas y se considera un hombre libre. La situación del esclavo es análoga a la situación de quien no puede distinguir entre el sueño y la vigilia ${ }^{33}$, más específicamente, alguien que cree que está despierta cuando en realidad está dormida. La persona que está soñando cree, durante el sueño, que mueve su cuerpo en determinada dirección (supone en sí cierto poder semoviente que le permite desplazarse espacialmente); sin embargo, esto no se condice con lo que de hecho ocurre, ya que su cuerpo, en realidad, no se está moviendo sino que yace en reposo.

El esclavo sería aquel que no se da cuenta de cómo afectan las pasiones a su alma. Es aquel que cree que está decidiendo racional o autónomamente realizar una acción, cuando, en realidad, esa decisión solo tiene una apariencia de racionalidad que no es más que una racionalización de lo que las pasiones lo han llevado a hacer. La experiencia consciente del esclavo sería engañosa, ilusoria, ya que habría fuerzas operando en su espíritu de las que él no tiene idea. En este sentido, el esclavo tampoco posee un criterio demarcador firme para distinguir entre experiencias engañosas y no-engañosas, como sucede con el sueño y la vigilia en el argumento cartesiano.

En su respuesta al fatalista, Du Châtelet parecería rechazar la posibilidad de que las pasiones actúen subrepticiamente en nuestro espíritu de forma permanente. Si bien la autora concede que algunas pasiones pueden tener un imperio temporal sobre nosotros, según ella, el hecho de que nos percatamos de tales momentos de esclavitud indica que alguna vez fuimos libres. En este sentido, para ella, la libertad es un poder que en algunas circunstancias puede activarse y en otras no. Desde el punto de vista de Du Châtelet, la libertad es la salud del alma; es débil, limitada, intermitente, nunca es plena. Nuestra libertad puede potenciarse, de acuerdo con la filósofa, mediante la costumbre adquirida de reflexionar antes de actuar. Sin embargo, aunque nos esforcemos, nunca podremos deshacernos de las pasiones, ya que serían movimientos involuntarios del alma.

\footnotetext{
30 DESCARTES, Pasiones del alma (I \$25), p. 74 (AT 11: 347).

31 DESCARTES, Pasiones del alma (II S52), p. 100 (AT 11: 372).

32 DESCARTES, Pasiones del alma I \$45-50. Tal como explica Shapiro: "[In the Passions of the soul] Descartes thinks that in regulating the passions we can change the naturally instituted associations between body and mind. This position suggests a subtle shift from that articulated in the Sixth Meditation. There, Descartes intimates that the associations governing mind-body interaction are law-like; they are instituted by God and so are at least contingently necessary, i.e., necessary given God's will. In the Passions Descartes moves away from this claim" (SHAPIRO, "Descartes's Passions of the Soup', p. 272).

33 Al finalizar la "Primera meditación", el fatigado meditador confiesa no poder sostener ese estado de duda radical que ha logrado en sus ejercicios meditativos: "Cierta desidia me arrastra insensiblemente hacia mi manera ordinaria de vivir; y, como un esclavo que goza en sueños de una libertad imaginaria, en cuanto empieza a sospechar que su libertad no es sino un sueño, teme despertar y conspira con esas gratas ilusiones para gozar más largamente de su engaño, así yo recaigo insensiblemente en mis antiguas opiniones" (DESCARTES, Meditaciones, pp. 124-125 [AT 7: 23]).
} 


\section{E1 segundo argumento}

En el segundo argumento, el fatalista invocado por Du Châtelet sostiene que aun cuando acordemos que tenemos un sentimiento interno de libertad, este es producto de un engaño sistemático. Aquí el fatalista se refiere a otro tipo de engaño, diferente al del primer argumento, ya que implica, además de la imposibilidad de discernir entre ilusión y realidad, una naturaleza humana en sí misma defectuosa, diseñada para fallar. Esta objeción fatalista describe el engaño acerca del sentimiento de libertad de la siguiente manera: "Vuestro sentimiento os engaña acerca de vuestra libertad, de la misma manera que vuestros ojos os engañan acerca del tamaño del sol cuando os hacen juzgar que el disco de este astro es de casi dos pies de largo, aunque su diámetro sea realmente respecto del de la Tierra aproximadamente como 100 es a $1{ }^{\prime \prime 34}$.

Podríamos observar aquí una referencia al argumento de los sentidos de Descartes y específicamente a la discrepancia que él plantea en la Tercera Meditación entre la idea de sol que proviene de los sentidos y la que proviene de los razonamientos de la astronomía. Du Châtelet interpreta que la objeción del fatalista asume este "engaño de la vista" como si nuestros órganos de los sentidos estuviesen diseñados para engañarnos sistemáticamente acerca de lo que percibimos. Entonces no se trata de un error ocasional sino de un defecto del aparato perceptual que se debe a cómo nos hizo Dios. Por eso, para responderle al fatalista, Du Châtelet alude a las leyes de la óptica y explica que, mediante el concurso de nuestro intelecto, podemos entender que existe una proporcionalidad entre el tamaño de los objetos percibidos y su distancia respecto del cuerpo del sujeto perceptor. Nuestros órganos sensoriales han sido diseñados para poder percibir los objetos más o menos cerca de nuestros cuerpos, según la filósofa. Por esta razón, si pudiésemos ver el tamaño real del sol, no nos servirían para ver ningún objeto sobre la Tierra. Así, la pensadora concluye que "Dios no me ha engañado al hacerme ver lo que está alejado de mí con un tamaño proporcional a su distancia" ${ }^{\prime 35}$.

Du Châtelet entiende que la analogía entre el ejemplo de la imagen del sol que trae el fatalista y el del sentimiento de libertad es inadecuada. Mientras que entre lo que veo y lo que juzgo que sea eso que veo puede haber una discrepancia salvable mediante el intelecto, según la filósofa, "nuestras acciones nos parecen libres precisamente de la misma manera que ellas nos lo parecerían si lo fuésemos verdaderamente" ${ }^{36}$. En nuestra percepción del sentimiento interior de libertad tal discrepancia no existe, de acuerdo con Du Châtelet, ya que este sentimiento consistiría en una [auto] percatación de nuestra propia actividad mental, cuando, por ejemplo, elegimos imaginar algo, y también de la ejecución de la acción cuando esta implica el movimiento del cuerpo propio.

Ella deduce que si me engaño acerca de este sentimiento interior (que se me aparece con un grado supino de certeza), entonces debería suponer que Dios me engaña. Más aún,

\footnotetext{
34 "Votre sentiment [interieur] vous trompe sur votre liberté, de même que vos yeux vous trompent sur la grandeur du soleil lorsqu'ils vous font juger que le [son] disque de cet astre est environ large de deux pieds, quoique son diametre soit reellement a celui de la terre environ comme 100 est a 1" (WADE, Studies, p. 96).

35“'Dieu ne m'a point trompé en me faisant voir ce qui est éloigné de moi d'une grandeur proportionnée à sa distance" (WADE, Studies, p. 97).

${ }^{36 " N o s}$ actions nous paroissent libres précisément de la même manière qu'elles nous le paroitroient si nous l'etions veritablement (note 4)" (WADE, Studies, p. 97).
} 
me habría creado para mantenerme en esta ilusión perpetua. Así, ella nos remite al argumento cartesiano del Dios engañador: puede que exista un Dios que me engañe inclusive acerca de las verdades matemáticas (las cuales el argumento del sueño no llega a poner en duda). "Ignoramos si por ventura [Dios] nos ha querido crear tales que nos engañáramos siempre, aun en aquellas cosas que nos parecen evidentísimas" ${ }^{37}$. Pero para Du Châtelet, la hipótesis de un Dios engañador es un absurdo, pues Dios no puede sino ser infinitamente sabio y veraz. Como el sentimiento que tenemos de nuestra libertad es tan fuerte, para desautorizarlo haría falta, según Du Châtelet, una demostración que comprobase que el ser libres implica una contradicción. Pero no la hay, de acuerdo con la autora.

\section{La nota 4}

Al finalizar la reconstrucción de la segunda objeción fatalista, se agrega una nota explicativa al texto (la número 4) que dice: "La respuesta a esta segunda objeción es casi la misma que la del tercer argumento [del capítulo precedente] contra la existencia de los cuerpos, pero esto no puede ser de otra manera porque las personas que niegan la libertad realizan contra ella [prácticamente] [las mismas] una parte de las objeciones que aquellos que niegan la existencia de los cuerpos hacen contra esta existencia"38. Habría entonces un solapamiento (al menos parcial) entre las objeciones que plantean quienes niegan la libertad y las de quienes ponen en duda la existencia de los cuerpos. Ambas podrían provenir de una matriz de pensamiento pirrónica, en donde el ejercicio de la duda se aplica incluso a ciertas tesis que damos por sentadas en nuestra vida cotidiana, como la de la libertad o la de la existencia de los cuerpos ${ }^{39}$.

En cuanto al capítulo precedente que se menciona en la nota, es menester señalar que en el capítulo IV del Traité de métaphysique se analizan los argumentos escépticos que nos hacen dudar respecto de la existencia de los cuerpos ${ }^{40}$. Si el manuscrito del DL fue reutilizado en algún momento del proceso de escritura como borrador del “capítulo V” [luego VII] del Traité, entonces la nota podría estar refiriéndose al capítulo IV de esta obra, obra que, recordemos, fue escrita por Voltaire y por Du Châtelet. Como han mostrado Kölving y Brown $^{41}$, la filósofa tuvo en ella una participación activa como co-autora y como revisora

\footnotetext{
${ }^{37}$ DESCARTES, Principios I $₫ 5$, p. 8 (AT 8: 6).

38 "La reponse a cette seconde objection est presque la meme que celle du $3 \mathrm{e}$ argument [du chapitre precedent] contre lexistence des corps mais cela ne peut etre autrement puisque les personnes qui nient la liberte font contrelle [a peu pres] [les memes] une partie des objections que ceux qui nient lexistence des corps font contre cette existence". En la transcripción que realiza Wade constatamos una aclaración a esta nota que indica: "(Nota de Mme du Châtelet)" (WADE, Studies, p. 97).

${ }^{39}$ La séptima nota que Du Châtelet toma de los Principios de Descartes nos demuestra que el problema de la existencia de los cuerpos estaba en el horizonte de reflexión de esta pensadora en relación con la obra del filósofo. La nota dice: "No sé si él [Descartes] tiene razón en la distinción que hace entre la longitud y el color, etc. Hablando metafísicamente, ¿no contradice esto la posibilidad de la no existencia de los cuerpos y el atomismo? Página 55". "Je ne sais si il a raison dans la distinction qu'il fait de la longueur \& et de la couleur \&c. Metaphisiquement parlant, cela ne contredit-il point la possibilité de la non existence des cors et l'atomisme page 55”.

KÖLVING, BROWN, ERTZ, "Three notebook leaves on Descartes, Newton, Fontenelle", [f. 122v] https://historyofwomenphilosophers.org/stp/documents/view/notes\#marker7

40 Para mayor detalle sobre estos argumentos en el Traité de métaphysique, véase BRANDÃO, "Voltaire et le scepticisme".

${ }^{41}$ Cf. BROWN, A. \& KÖLVING, U. “Qui est l'auteur du Traité de métaphysique?”. In : Cahiers Voltaire, n. 2, pp. 85-94, 2003.
} 
crítica. Gardiner Janik ${ }^{42}$, por su parte, argumenta que en la versión temprana de las IP habría existido un capítulo en el que se examinaban razones a favor y en contra de la existencia de los cuerpos que fue, junto con otros, o no incluido en la obra o radicalmente reescrito para expresar doctrinas wolffianas sobre los elementos constitutivos de la realidad y la materia ${ }^{43}$.

Si bien Descartes no es el único interlocutor polémico de Du Châtelet en DL, su particular empleo de argumentos escépticos reviste un peligro epistemológico que la filósofa se siente compelida a enfrentar — tanto en DL como en las IP_. Esta batería de argumentos cartesianos tiene como objetivo poner en duda nuestra confianza en las facultades cognitivas (aunque más no sea momentáneamente). En tanto no sean resueltos o disueltos, podrían socavar los fundamentos de la investigación científica.

Así, los argumentos escépticos de las objeciones fatalistas estudiadas cuestionan el recto funcionamiento de nuestras facultades cognitivas respecto del "sentimiento interior de libertad” que experimentamos. Lo que está en juego en esta discusión no es únicamente si somos o no somos libres sino si existe algún principio que nos permita garantizar que no nos engañamos en el uso de nuestras facultades con respecto de nuestra [presunta] libertad. Esta preocupación repercute en las IP. Allí, la autora se esfuerza por demostrar que el precepto metodológico cartesiano de solo aceptar como verdaderas aquellas ideas claras y distintas (para luego basar en ellas subsiguientes razonamientos) no es adecuado como fundamento epistémico del conocimiento científico. Du Châtelet entiende que el "sentimiento vivo e interno" 44 de claridad y evidencia posee un carácter puramente subjetivo y, por lo tanto, se presta a generar confrontaciones irresolubles entre adversarios intelectuales.

\section{Du Châtelet contra Descartes en Institutions de Physique (o cómo blindarse contra ataques pirrónicos)}

Uno de los primeros objetivos que Du Châtelet se propone cumplir en las IP consiste en definir un marco metodológico unificado para su proyecto filosófico-científico. Descartes constituye, en este sentido, una figura de la que Du Châtelet deberá distanciarse para dar por tierra con el subjetivismo cartesiano y blindarse contra posibles ataques pirrónicos ${ }^{45}$. Para la filósofa, el criterio cartesiano según el cual cada sujeto pensante debería tomar por verdadero todo aquello que se le presenta como claro y distinto a su espíritu

no sirve sino para eternizar las disputas, porque quienes tienen sentimientos opuestos tienen cada uno este sentimiento vivo e interno de la verdad de lo que proponen; así ninguno debe de rendirse, en tanto la evidencia es igual de

\footnotetext{
42 GARDINER JANIK, "Searching for the Metaphysics of Science...”, pp. 100-101.

${ }^{43}$ Según Anne-Lise Rey, una de las razones por las cuales Du Châtelet finalmente no incluye el DL en las IP es que en esta última "elle renonce à une conception atomiste de la matière au profit de la reconnaissance d'une élasticité essentielle aux corps". REY, "La Minerve vient de faire sa physique", p. 242. Puede que este cambio haya incidido en el destino del capítulo sobre la existencia o inexistencia de los cuerpos.

$44 \mathrm{IP} \int 2$, p. 17.

45 A pesar de sus críticas, Du Châtelet profesa una gran admiración por Descartes, la cual expresa en l'Avantpropos de sus IP. Tal como sostiene Detlefsen en su artículo "Émilie du Châtelet" de la Stanford Encyclopedia of Philosophy, las IP de Du Châtelet emulan en cierta medida la estructura de los Principios de Descartes (en los cuales el filósofo también busca defenderse del pirronismo), si bien existen grandes divergencias entre ambas obras.
} 
los dos lados. Es necesario entonces sustituir por demostraciones las ilusiones de nuestra imaginación y no admitir nada como verdadero, salvo lo que puede deducirse, de manera incontestable, de los primeros principios que nadie puede poner en duda y rechazar como falso todo aquello que es contrario a estos principios o a las verdades que se establecen por este medio, sea lo que sea que pueda decir la imaginación ${ }^{46}$.

De acuerdo con Du Châtelet, el subjetivismo solipsista llevaría a cierto estado de isosthéneia, es decir, a cierto estado de equivalencia de las razones o las justificaciones al que buscan arribar los pirrónicos mediante sus tropos para neutralizar las pretensiones de verdad del dogmático, logrando la suspensión del juicio. Determinar cuál de dos tesis opuestas es verdadera y cuál es falsa se vuelve una tarea inasequible si la justificación de estas tesis se basa en un sentimiento interior de quien la defiende. En tanto cada uno/a de los/as rivales tiene un acceso privilegiado y personal a sus propios estados mentales (corolario del solipsismo cartesiano), no hay forma de validar o invalidar externamente las tesis en cuestión. La convicción que aporta el sentimiento vivo e íntimo no es suficiente entonces en las explicaciones filosóficas y científicas para Du Châtelet. Se debe llegar a un criterio de verdad incontestable para todos/as basado en principios indubitables e inferencias deductivas a partir de estos que evite el peligro de esta contienda infinita e imposible de dirimir que plantea el escepticismo. Estos principios incontestables, que nadie puede poner en duda, son, para Du Châtelet, el principio de contradicción y el principio de razón suficiente.

Respecto del primero de ellos, Du Châtelet entiende que es el primer axioma sobre el que se fundan todas las verdades necesarias (IP \$4) y explica que "los pirrónicos mismos, que hacen profesión de dudar de todo, nunca han negado este principio" 47 . Adicionalmente, ella encuentra en este principio la clave para demostrar la verdad del cogito ergo sum cartesiano (IP \$7). En efecto, Du Châtelet argumenta que afirmar que una tiene una idea, por ejemplo, una duda (de si existe o no), y sostener al mismo tiempo que una no existe es contradictorio. Por eso, quien duda acerca de si existe o no posee en esa duda una prueba de su existencia.

El principio de razón suficiente es aquel que debe aplicarse a las verdades contingentes, según la filósofa. Este principio da cuenta de las razones que nos permiten comprender por qué una cosa es de cierta manera y no de otra ${ }^{48}$. Resulta particularmente relevante uno de los ejemplos que Du Châtelet utiliza para ilustrar las absurdidades a las que llegamos en nuestras reflexiones cuando suponemos que las cosas pueden suceder sin una razón suficiente. Una de estas absurdidades consiste en no poder discernir entre el mundo fabuloso de los sueños y el de la vigilia. En IP $\$ 8$, la pensadora se enfrenta al argumento escéptico del sueño y pretende resolverlo mediante el principio de razón suficiente.

\footnotetext{
46 “Cette méthode, d'ailleurs, ne serviroit qu’à éterniser les disputes, car ceux qui ont des sentimens opposés, ont chacun ce sentiment vif \& interne de la vérité de ce qu'ils avancent; ainsi aucun ne doit se rendre, puisque l'évidence est égale des deux côtés; il faut donc substituer des démonstrations aux illusions de notre imagination, $\&$ ne rien admettre comme vrai, que ce qui peut se déduire, d'une manière incontestable, des prémiers principes que personne ne peut révoquer en doute \& rejetter comme faux tout ce qui est contraire à ces principes, ou aux vérités que l'on a établies par leur moyen, quoiqu'en puisse dire l'imagination" (IP \$2, p. 18).

47 "Les Pirrhoniens mêmes qui faisoient profession de douter de tout, n'ont jamais nié ce principe" (IP, p. 19).

${ }^{48}$ Para un análisis de las diferencias entre Du Châtelet y Leibniz en torno al principio de razón suficiente, véase la conferencia dictada por Fatema AMIJEE, "The disharmony between Leibniz and Du Châtelet", en el seminario VAMP (Vanderbilt Modern Philosophy): https://www.youtube.com/watch?v=n_LxASdNbD4.
} 
Lo que ocurre a veces en sueños nos da la idea de un mundo fabuloso, donde todos los eventos suceden sin razón suficiente. Sueño que estoy en mi habitación, ocupada escribiendo; de pronto, mi silla se transforma en un caballo alado y me encuentro en un instante a cien leguas del lugar en donde estaba y con personas que hace tiempo han muerto. Todo eso no puede ocurrir en este mundo, porque no habría razón suficiente de todos esos efectos; pues cuando salgo de mi habitación, puedo decir cómo y por qué salgo de ella y no voy a otra sin pasar por lugares intermedios. Sin embargo, todas estas quimeras serían igualmente posibles si pudiese haber efectos sin razón suficiente: este es el principio que distingue el sueño de la vigilia y el mundo real del mundo fabuloso que nos pintan los cuentos de hadas ${ }^{49}$.

Vemos, de esta manera, que la secuencia de causas y efectos que percibimos mientras estamos despiertos/as puede explicarse apelando a este principio. Sin embargo, en un mundo quimérico o ilusorio como el de los sueños, nuestras representaciones se siguen unas a otras de forma arbitraria, espontánea o azarosa. Du Châtelet ofrece, de esta forma, un principio que oficia de criterio para distinguir entre la vigilia y el sueño.

\section{Conclusión}

A lo largo de este artículo, hemos intentado mostrar el recorrido intelectual temprano de Du Châtelet en su ponderación del tema de la libertad como poder semoviente y en su combate contra el pirronismo. Consideramos que ambos se unen y se amalgaman en DL, en donde la filósofa recrea distintas objeciones fatalistas para rebatirlas y gestar así su propia posición al respecto. Se integra de esta forma en un debate que, tal como demuestra la riqueza de referencias a otros/as pensadores/as modernos/as en DL, apasionó a los/as filósofos/as de la época.

Al describir la consolidación de la posición metodológica y epistemológica de la autora en las IP, constatamos su rechazo de la idea cartesiana de que un "sentimiento vivo interno" pueda conformar una base segura para la fundamentación de nuestros conocimientos. Sin embargo, es menester tener en cuenta a su vez una característica relevante de su posicionamiento metodológico que se transforma en la transición del DL a las IP. Recordemos que, en su respuesta al segundo argumento fatalista en DL, Du Châtelet recurre a la definición de Dios como ser infinitamente veraz y bondadoso para argumentar que es imposible que este nos engañe. Esta problemática apelación a Dios signará el destino del texto, el cual culmina de una forma aporética. Hacia el final, Du Châtelet confiesa que ella es incapaz de explicar cómo es que la libertad humana puede ser compatible con la omnisciencia

\footnotetext{
49 "Ce qui arrive quelquefois en songe nous fournit l'idée d'un monde fabuleux, où tous les évènemens arriveroient sans raison suffisante. Je rêve que je suis dans ma chambre, occupée à écrire ; tout d'un coup ma chaise se change en un cheval ailé, \& je me trouve en un instant à cent lieues de l'endroit où j'étois, \& avec des personnes qui sont mortes depuis longtems, \&c. Tout cela ne peut arriver dans ce monde, puisqu'il n'y auroit point de raison suffisante de tous ces effets; car lorsque je sors de ma chambre, je puis dire comment, \& pourquoi j'en sors, \& je ne vais point d'un lieu dans un autre sans passer par les lieux intermédiaires: cependant toutes ces chimères seroient également possibles, s'il pouvoit y avoir des effets sans raison suffisante: c'est ce principe qui distingue le songe de la veille, \& le monde réel, du monde fabuleux que l'on nous dépeint dans les Contes des Fées" (IP, pp. 25-26).
} 
divina. Confesión que acompaña con un pronunciamiento acerca de los límites de la libertad humana. Estos límites se dan de distinta manera en todas las criaturas de la Naturaleza e incluso, dentro de la especie humana, entre niños y adultos.

Estos pasajes habrían resultado quizás inadecuados o desmotivadores en relación con el ambicioso proyecto filosófico-científico de las IP, y con relación al poder de la razón humana que debe suponerse para desarrollarlo. Uno de los motivos por los cuales Du Châtelet podría haber eliminado este capítulo y en general modificado tan radicalmente toda la obra de las IP reside en que, para la autora, tal empresa de fundamentación metafísica del cosmos no puede sostenerse mediante apelaciones a Dios como recurso explicativo de ciertos fenómenos físicos y metafísicos para los que no se encuentra otra explicación.

El DL anuncia a su vez muchas de las preocupaciones ético-políticas que Du Châtelet seguirá explorando en obras posteriores como, por ejemplo, el Discurso sobre la felicidad. Si hay algo que nos demuestra el DL es que esas escrituras abandonadas, tachadas, marginales, esos bosquejos que los/as autores/as deciden no exhibir públicamente, a menudo resultan valiosos registros, particularmente en el marco de la labor de recuperación y de valorización del trabajo filosófico de una figura que ha sido tradicionalmente relegada en la disciplina hasta hace algunas pocas décadas como Émilie Du Châtelet.

\section{Referencias bibliográficas}

\section{Fuentes}

DU CHÂTELET, E. Institutions de Physique, manuscrito autógrafo, BNF, Fr, 12265, 1737. Institutions physiques. Amsterdam: Aux depens de la compagnie, 1742.

Les Lettres de la Marquise Du Châtelet. 2 Vols. Besterman, T. (Ed.). Geneva: Institut et Musée Voltaire, 1958.

La Correspondance d'Émilie du Châtelet. Brown, A. e Kölving, U. (Eds.). Paris: Centre international d'étude du XVIIIe siècle, 2018.

WADE, I. Studies on Voltaire. With Some Unpublished Papers of Mme du Châtelet. Princeton: Princeton Univ. Press, 1947.

\section{Complementaria}

BERNSTEIN, M. "Fatalism". In: The Oxford bandbook of freewill. Kane, R. (Ed.). Oxford: Oxford University Press, 2002.

BRANDÃO, R. "Voltaire et le scepticisme”. In: Philosophiques, n. 35 (1), pp. 261-274, 2008. https://doi.org/10.7202/018249ar 
BROWN, A. \& KÖLVING, U. "Qui est l'auteur du Traité de métaphysique?”. In : Cahiers Voltaire, n. 2, pp. 85-94, 2003.

CAUSSY, F. Inventaire des manuscrits de la Bibliothèque de Voltaire conservés à la Bibliothèque Impériale de Saint-Pétersbourg. Paris: Imprimerie nationale, 1913.

CHARLES, S. "O solipsismo como forma extrema de ceticismo no Século das Luzes". In: Doispontos n. 2 (4), pp. 13-38, 2007.

CROUSAZ, J.-P. Examen du pyrrbonisme ancien et moderne. La Haya: Pierre de Hondt, 1733.

DESCARTES, R. CEuvres de Descartes, 12 vols. Adam, C. e Tannery, P. (Eds.). Paris: Vrin, 1895-1910. 1977. Meditaciones metafísicas con objecionesy respuestas, Trad. de Vidal Peña. Madrid: Alfaguara, Los principios de la filosofía. Trad. de Gregorio Halperín. Buenos Aires: Losada, 1997. Las pasiones del alma. Trad. de Tomás Onaindia, Introducción de Agustín Izquierdo. Madrid: EDAF, 2005.

DETLEFSEN, K. "Émilie du Châtelet". In: Zalta, E. (Ed.), The Stanford Encyclopedia of Philosophy, 2018. https://plato.stanford.edu/archives/win2018/entries/emilie-du-chatelet.

GARDINER JANIK, L. "Searching for the Metaphysics of Science: The Structure and Composition of Madame du Châtelet's Institutions de physique, 1737-1740". In: Studies on Voltaire and the Eighteenth Century, n. 201, pp. 85-113, 1982.

HAGENGRUBER, R. "If I were king! Morals and Physics in Emilie Du Châtelet's Subtle Thoughts on Liberty". In: Women and Liberty, 1600-1800: Philosophical Essays, Broad, J., e Detlefsen, K. (Comps.). Oxford: Oxford Scholarship Online, 2017.

HATFIELD, G. "Transparency of Mind: The Contributions of Descartes, Leibniz, and Berkeley to the Genesis of the Modern Subject". In: Departure for Modern Europe: A Handbook of Early Modern Philosophy (1400-1700), Busche, H. (Ed.). Hamburg: Felix Meiner Verlag, 2011.

JORATI, J. "Du Châtelet on freedom, self-motion, and moral necessity". In: Journal of the History of Philosophy, n. 57 (2), pp. 255-80, 2019.

MASON, H. Voltaire. Barcelona: Salvat, 1986.

PAILLARD, C. "Entre science et métaphysique: le problème du fatalisme dans la philosophie de Voltaire”. In: Revue Voltaire, n. 8, pp. 207-223, 2008.

POPKIN, R. The history of scepticism from Savonarola to Bayle. Oxford: Oxford University Press, 2003. 
REY, A.-L. “La Minerve vient de faire sa physique”. In: Philosophiques, n. 44 (2), pp. 233-253, 2017. https://doi.org/10.7202/1042332ar

SHAPIRO, L. "Descartes's Passions of the Soup'. In: Philosophy Compass, n. 1 (3), pp. 268-278, 2006.

ZANCONATO, A. La dispute du fatalisme en France: 1730-1760. Paris: Presses Paris Sorbonne, 2004.

ZINSSER, J. La Dame d'Esprit: A Biography of the Marquise Du Chatelet. New York: Penguin, 2006. 